\title{
Single stage Large LR recession with or without MR resection and its motor outcomes in Concomitant Exotropia
}

Nalini Jayanthi B ${ }^{1}$, Y Raman ${ }^{2}$, N Sunitha ${ }^{3}$

1. Assistant Professor of Ophthalmology,Government Medical College, Nalgonda

2. Professor of ophthalmology,Department of pediatric ophthalmology and neuroophthalmology. Sarojini Devi Eye Hospital,Hyderabad.

3. Civil Assistant surgeon,Sarojini Devi Eye Hospital,Hyderabad

Corresponding Author: Dr.Y.Raman, M.S., Professor of ophthalmology, Department of pediatric ophthalmology and neuroophthalmology. Sarojini Devi Eye Hospital, Hyderabad.

DOI: 10.47799/pimr.0803.06

(C) 2020-21 Prathima Institue of Medical Sciences

\section{INTRODUCTION}

Exotropia is a manifest outward deviation of the visual axes, which is either constantly or intermittently present. Untreated, poorly controlled intermittent exotropia later progresses to constant exotropia.

Sensory exotropia is unilateral divergent misalignment of the eyes, resulting from loss of vision or long-standing poor vision in an eye.

In sensory exotropia the angles are characteristically large, ranging from 30 to 100 prism dioptres (PD) and increases gradually over time as long as the cause of visual deficit remains active. Treatment is directed to re-establish the normal ocular alignment and binocular vision ${ }^{[1]}$. The preferred treatment for manifest exotropia is surgery ${ }^{[2]}$. Large angle constant exotropia negatively impacts the way the patients see themselves and are perceived by others. The benefits of surgical treatment of exotropia in adults is well proven, both psychologically and visually. The surgical treatment for largeangle exotropia has been a subject of sufficient debate. A variety of surgical plans have been described including two, three or four horizontal rectus muscles recession and resections with or without adjustable suture technique. ${ }^{[3]}$ In more recent studies, the management of large-angle exotropia falls into two surgical approaches.

Large bilateral lateral rectus recession is done when the acuity is good in each eye and indicated for true divergent excess type. For basic exodeviation R-R procedure is done popularly. Before embarking on surgical plan we do post occlusion test and identify the clinical type.

If one eye is amblyopic, a maximal or supramaximal unilateral recess- resect procedure is performed.

Records of previous studies have shown that monocular surgery had shown good results for exotropia of $<60$ PD. But for exotropia of $>60$ PD, monocular surgery was not so effective (4).

In previous studies mild to moderate LR recessions were described but our study involves maximum recession on LR. In previous studies large LR recessions were reported to have complications like Lid changes and palpebral aperture widening.
Submission : 16-10-2020

Review : 20-10-2020

Acceptance 21-10-2020
This study was done to evaluate the outcome of single stage, unilateral large LR recession with or without MR resection for constant exotropia.

\section{METHODS :}

Our study included 51 consecutive patients with exotropia ranging 35 to $>100 P D$, who underwent unilateral single stage large $L R$ recession with or without $M R$ resection from September 2018 to July 2019 in tertiary eye hospital. Approval taken from ethics committee of the hospital.

The exclusion criteria were Exodeviation of <35PD, Paralytic squint and any history of previous squint surgery.

In all the patients, a detailed history was taken regarding age of onset of squint, family history, whether intermittent or constant, history of diplopia, ocular trauma or any previous surgery.

All patients underwent complete ocular examination before surgery. Visual acuity was measured using Snellen chart, E chart or broken ring chart with and without glasses.

The ocular alignment was assessed with the help of cover/ uncover test. Amount of deviation were measured by alternate prism bar cover test, both for distance and near, majority of cases showed basic type which were managed by resect and recess procedure.

Slit lamp examination was done for every patient to rule out any anterior segment pathology. Intraocular pressures were measured using Goldmann Applanation tonometry. Posterior segment was also evaluated by direct ophthalmoscopy and slit lamp biomicroscopy using $90 \mathrm{D}$ lens to rule out any pathology.

Patients under 16 years of age were taken under general anesthesia and above 16 years operated under local anesthesia.

Muscles were exposed by limbal based conjunctival flap and hang back technique used for recession of the LR muscle.

Maximum surgical handling (LR recession) was done in order to spare the other muscles in case of recurrence.

Patients with exotropia upto 45 to 50 PD underwent unilateral large LR recession. Patients with $\mathrm{XT}$ of $>60 \mathrm{PD}$ underwent unilateral large $L R$ recession combined with $M R$ resection.

The postoperative follow up were done at day 1,1 week, 1 
month, 3 months and 6 months. In all the follow-ups, ocular alignments were assessed with the help of cover/ uncover test. Any amount of overcorrection or under correction were measured by alternate prism bar cover test.

After completing the follow up the data collected and statistical analysis done. Statistical analysis carried using SPSS 12 version particularly mean, standard deviation and Paired t-test calculated.

\section{RESULTS}

The study included 51 patients with exotropia ranging from 35 to 100 PD, who underwent unilateral single stage large LR recession with or without MR resection. Twenty eight patients (54.97\%) were males and Twenty three patients (45.1\%) were females. Mean age of the patients were (21.22) years (range 5 - 48 years). The mean preoperative deviations at were (40$>100$ PD) $(59.16+16.212)$. Mean post OP deviation $(4.43+$ 2.77). None had intra operative complications related to surgery or Anesthesia. None of the patients had limitation of ocular movement and any lateral incomitance.

In all patients, unilateral large LR recession with or without $M R$ resection was done in single stage. Mean recession 10.54MM and range is (10-12MM). Mean resection 6.25MM (5 to $8.5 \mathrm{MM}$ ). The postoperative result showed that 36 patients $(70.0 \%)$ had orthotropia two patients $(3.9 \%)$ had mild esodeviation and eight patients (15.6\%) had residual exo and one patient (1.5\%) had to undergo release of adhesion. Thus, forty six patients (88.\%) in our study had good cosmetically acceptable outcome. Only one patient had to undergo release of adhesions as a second surgery.

None of the patients in our study had any significant limitation of ocular movement after surgery. $50 \%$ of the patients complained of Diplopia which resolved over a period of 2-3 weeks. None of the patients showed any lid changes or widening of the palpebral aperture.

Statistical analysis carried using SPSS 12 version particularly Mean, Standard deviation and Paired t- test calculated. The calculated t- value 25.629 and corresponding $p-0.000$ the statistically significant both pre OP and post OP tests at 0.05 level of significance. The statistical analysis shows significant post OP correction of the deviation.

As per gender and age concerned, pertaining to surgical outcome there is no significant intergroup difference.

Figure1

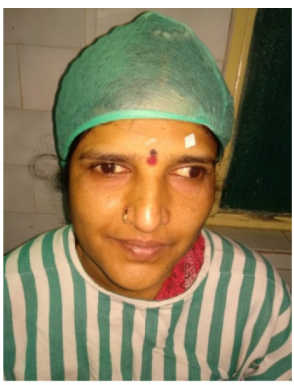

Primary gaze

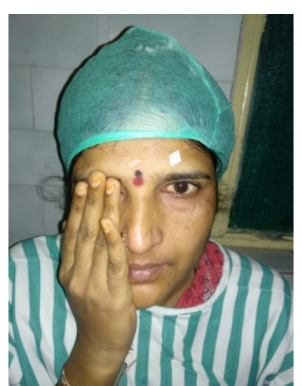

With cover test
Figure2

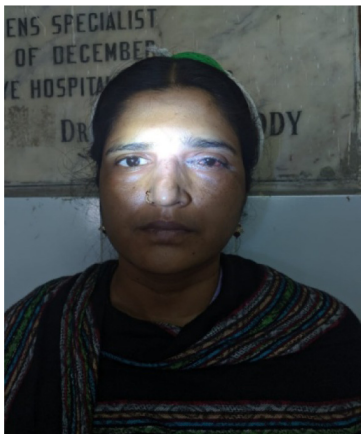

$1^{\text {st }}$ Post OP day

Table-1

\begin{tabular}{|l|l|l|}
\hline & No. of Patients & Percentage \\
\hline Male & & \\
\hline Female & & \\
\hline
\end{tabular}

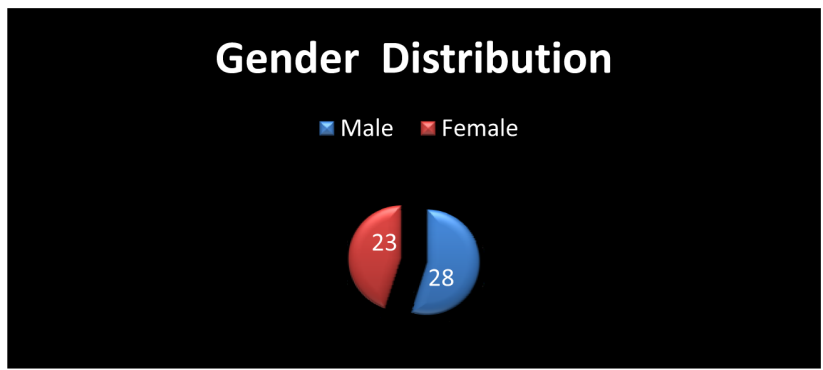

\begin{tabular}{|l|c|c|}
\hline \multicolumn{1}{|c|}{ Age } & No. of Patients & Percentage \\
\hline $1-10$ & 5 & $9.8 \%$ \\
\hline $11-20$ & 24 & $47.05 \%$ \\
\hline $21-30$ & 14 & $27.44 \%$ \\
\hline $31-40$ & 2 & $3.9 \%$ \\
\hline $41-50$ & 6 & $11 \%$ \\
\hline
\end{tabular}

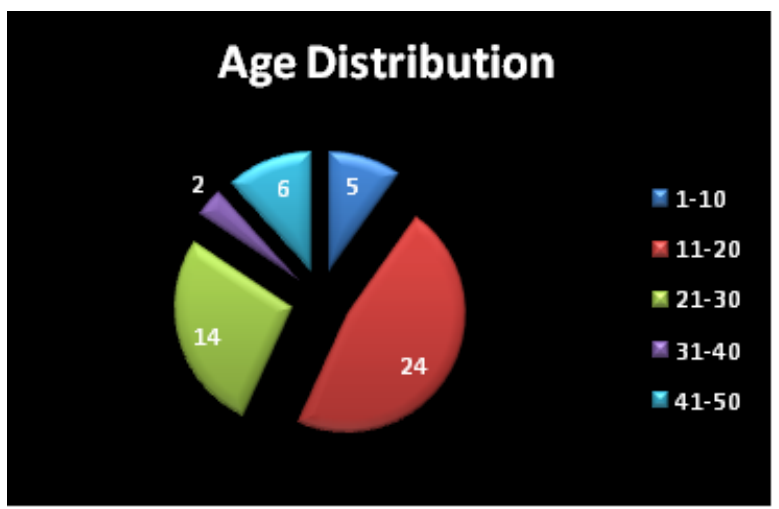


Table-3

\begin{tabular}{|l|c|c|}
\hline \multicolumn{1}{|c|}{ Deviations } & No. of Patients & Percentage \\
\hline Upto 50PD & 22 & $45.21 \%$ \\
\hline 50-75 PD & 20 & $41 \%$ \\
\hline 75->100 PD & 9 & $14 \%$ \\
\hline
\end{tabular}

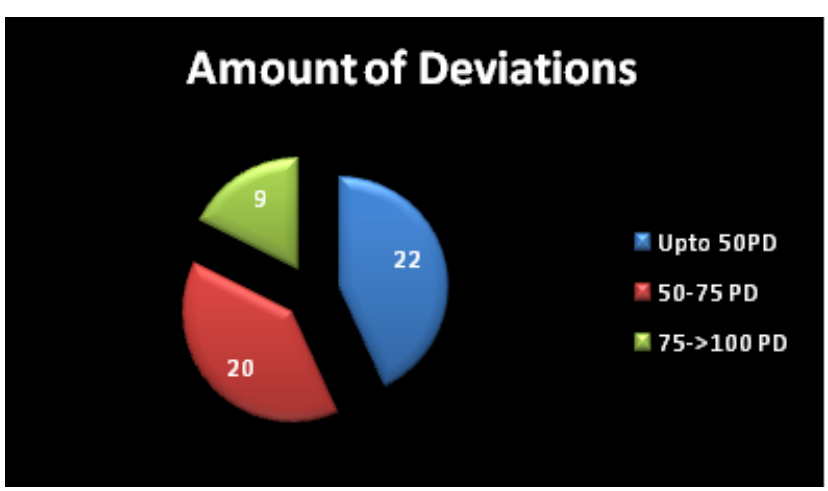

Table-4

\begin{tabular}{|l|c|c|}
\hline \multicolumn{1}{|c|}{ No. of Muscles } & No. of Patients & Percentage \\
\hline Single muscle (LR) & 8 & $15 \%$ \\
\hline Single eye (R\&R) & 39 & $76 \%$ \\
\hline Bilateral LR & 1 & $1.9 \%$ \\
\hline Three muscles & 2 & $3.9 \%$ \\
\hline
\end{tabular}

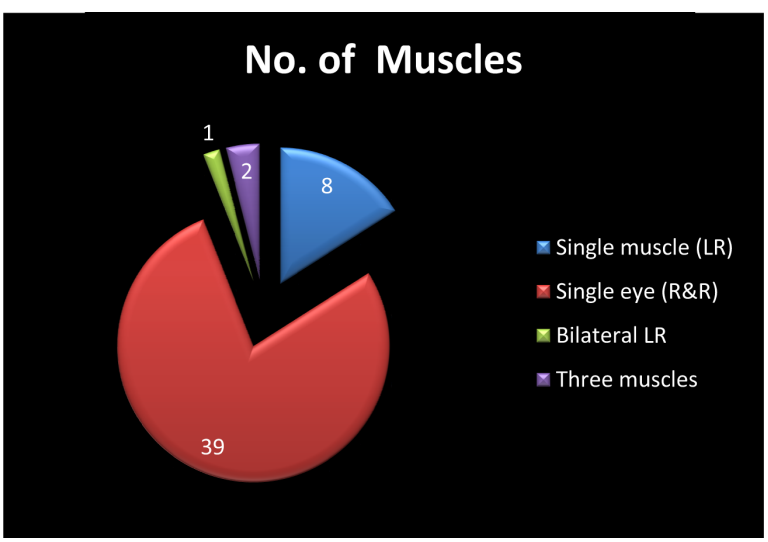

\section{DISCUSSION}

There have been various studies regarding the management of large-angle exotropia, both surgical and non-surgical. The surgical technique includes two, three- or four-muscle surgery done in one or two stages. ${ }^{[5-8]}$ It is advised by most authors that up to two-muscle surgery should be performed in one stage and if there is any exodeviation remaining, it should be corrected by a second stage surgery. ${ }^{[8]}$ According to Kushner et al, unilateral recession and resection procedure should be performed for primary exotropia. ${ }^{[9]}$
In our study, we have reviewed patients with exotropia of 35 to 100 PD who underwent single stage unilateral large LR recession with or without MR resection procedure. The added benefit of this procedure is that if in case a second operation is needed some muscles have been preserved for it. The postoperative result showed that 36 patients $(70.0 \%)$ had orthotropia two patients (3.9\%) had mild esodeviation and eight patients (15.6\%) had residual exo and one patient (1.5\%) had to undergo release of adhesion. Thus, forty six patients $(88 \%)$ in our study had good cosmetically acceptable outcome. Only one patient had to undergo release of adhesions as a second surgery.

The success rate of our study is similar to various previous studies done by other authors. For medium-angle exotropia two muscle surgery may be an option, while large-angle exotropia may need three or four muscle surgery. ${ }^{[10,11]}$

A report given by Ganguly et al ${ }^{[11]}$ showed a success rate of 83.3\% with unilateral two muscle surgery in exodeviation of 40 - 80 PD. In a study by Schwartz et al, ${ }^{[12]}$ two muscle surgery had good result for large-angle exotropia and $77 \%$ of their cases achieved a postoperative alignment of \pm 15 PD.

According to Gezer and Kushner, they identified that the most important factor to determine the successful outcome of the surgery in exotropia is the preoperative deviation. ${ }^{[13,14]}$. Thus, it has been accepted by many authors that preoperative deviation plays the most important role in determining the postoperative alignment. But there is no definite cut-off to bring about a favourable outcome with two muscles procedure, because other factors play a role as well such as surgical technique and dose. ${ }^{[10]}$

A study by Koo et al shows that the postoperative result does not depend on the age of operation. The favourable age for operation is when the child can be assessed for orthoptic correction and also when the functional and cosmetic symptoms become to apparent. ${ }^{[15-18]}$

According to the study conducted by Junejo SA et al, For medium-angle exotropia two muscle surgery may be an option, while large-angle exotropia may need three or four muscle surgery. ${ }^{[19]}$ the desired post op effect was seen with preoperative deviation of $<60$ PD. With deviation > 60 PD, there was difficulty in aligning the eyes with operation only on one eye. For these cases, a second surgery was required with more than two muscle procedure. ${ }^{[19]}$ In our study, one patient with exotropia of $>100$ PD required second surgery.

Although, it has been recommended by most authors not to exceed $7 \mathrm{~mm}$ medial rectus resection and $8 \mathrm{~mm}$ lateral rectus recession in order to avoid ocular movement limitations, ${ }^{[4,20,21]}$. In our study we have done lateral rectus recession of 10-12 $\mathrm{mm}$ and medial rectus resection of $5.5-8.5 \mathrm{~mm}$. None of the patients in our study had significant postoperative restriction of ocular motility, $50 \%$ of patients with diplopia resolved within 2 weeks and none of the patients showed any lid changes or widening of the palpebral aperture. 


\section{CONCLUSION}

Our study showed that single stage unilateral large LR recession with or without MR resection showed good result for exotropia of 35 to 100 PD. Also, large amount of lateral rectus recession and medial resection was safe and does not produce any significant postoperative motor deficit, lid changes and widening of the palpebral aperture.

\section{Acknowledgement}

We thank Dr.Srinivas J, for his constant help in preparing and presenting the statistical analysis.

\section{REFERENCES}

1. Eibschitz-TsimhoniM, Archer SM, Furr BA, et al. Current concepts in the management of concomitant exodeviations. ComprOphthalmol Update 2007;8(4):21323.

2. Asjes-Tydeman WL, Groenewoud H, Van der Wilt GJ. Timing of surgery for primary exotropia in children. Strabismus2006;14(4):191-7.

3. Yang $M$, Chen J, Shen T, et al. Single stage surgical outcomes for large angle intermittent exotropia. PLoS One2016;11(2):e0150508.

4. Millan T, de Carvalho KM,Minguini N. Results of monocular surgery under peribulbaranaesthesia for largeangle horizontal strabismus. Clinics (Sao Paulo) 2009;64(4):303-8.

5. Lau FHS, Fan DSP, Yip WWK, et al. Surgical outcome of single-staged three horizontal muscles squint surgery for extra-large angle exotropia. Eye (Lond) 2010;24(7):11716.

6. Mulberger RD, McDonald PR. Surgical management of nonparalytic exotropia. AMA Arch Ophthalmol 1954;52(5):664-8.

7. Aslanis D, Follidi V, Constantopoulos I, et al. Surgicalresultsin childhood primary comitant large-angle exotropia. J FrOphthalmol2006;29(1):37-42.

8. Thomas S, Guha S. Large-angle strabismus: can a single surgicalprocedure achieve a successful outcome? Strabismus2010;18(4):129-36.

9. Kushner BJ,Fisher MR, Lucchese NJ, et al. Factors influencingresponse to strabismus surgery. Arch Ophthalmol1993;111(1):75-9.

10. Saleem QA, Cheema AM, Tahir MA, et al. Outcome of unilateral lateral rectus recession and medial rectusresection inprimary exotropia. BMC Res Notes 2013;6:257.

11. Ganguly S, Pradhan R. Effect of monocular surgery forlarge-angle horizontal deviationin adults. Nepal J Ophthalmol2011;3(1):27-30.
12. SchwartzRL, Calhoun JH. Surgery of large angle exotropia.J PediatrOphthalmol Strabismus 1980;17(6):359-63.

13. Gezer A, Sezen F, Nasri N, et al. Factors influencing the outcomeof strabismus surgery in patients with exotropia. J AAPOS2004;8(1):56-60.

14. Kushner BJ. Selective surgery for intermittentexotropia based on distance/near differences. Arch Ophthalmol1998;116(3):324-8.

15. KooNK, Lee YC, Lee SY. Clinical study for the undercorrectionfactor in intermittent exotropia. Korean J Ophthalmol2006;20(3):182-7.

16. Richard JM, Parks MM. Intermittent exotropia. Surgical results in different age groups. Ophthalmology 1983;90(10):1172-7.

17. Keenan JM, Willshaw HE. The outcome of strabismussurgery in childhood exotropia. Eye 1994;8(Pt 6):632- 7 .

18. Stoller SH,Simon JW, Lininger LL. Bilateral lateral rectus recession for exotropia: a survival analysis. J PediatrOphthalmol Strabismus1994;31(2):89-92.

19. Junejo SA, Ansari MA. Outcome of monocular surgery for horizontal strabismus in Hyderabad. ClinOphthalmo 12010;4:269-73.

20. Damanakis AG, Arvanitis PG, Ladas ID, et al. $8 \mathrm{~mm}$ bimedial rectus recession in infantile esotropia of 80-90 prism dioptres. Br J Ophthalmol 1994;78(11):842-4.

21. Prieto-Díaz J, Souza-Dias C. Esotropias. In: Prieto-Díaz, Souza-Dias C. eds. Estrabismo. São Paulo: Livraria Santos Editora2002:149-99.

How to cite this article : BN Jayanthi, Y Raman, N Sunitha. Single stage Large LR recession with or without MR resection and its motor outcomes in Concomitant Exotropia. Perspectives in Medical Research 2020; 8 (3):25-28.

DOI: 10.47799/pimr.0803.06

Sources of Support: Nil, Conflict of interest: None declared 